18,12

\title{
О декорировании зигзагообразной кромки эпитаксиального графена
}

\author{
() С.Ю. Давыдов \\ Физико-технический институт им. А.Ф. Иофффе РАН, \\ Санкт-Петербург, Россия \\ E-mail: Sergei_Davydov@mail.ru \\ (Поступила в Редакцию 18 июня 2018 г. \\ В окончательной редакции 27 июня 2018 г.)
}

\begin{abstract}
Предложены два подхода к решению задачи о связи адсорбированных частиц с атомами зигзагообразной кромки графена, сформированного на металлической подложке. Первый подход базируется на схеме Калкстейна и Совена, позволяющей определить электронную структуру полубесконечного листа графена. В основе второго подхода лежит кластерная модель зигзагообразной кромки. Получены аналитические выражения для локальных плотностей состояний системы и чисел заполнения адатома углерода и адчастицы. Подробно рассмотрен случай изолированной адчастицы и предложен способ учета диполь-дипольного взаимодействия адчастиц, выстраивающихся вдоль кромки.
\end{abstract}

DOI: 10.21883/FTT.2019.01.46912.171

\section{1. Введение}

Нарастающий интерес к декорированию островков, или чешуек (flakes) графена, т.е. присоединению к их краям чужеродных частиц, вызван желанием управлять ростом, структурой и электронными характеристиками этих объектов [1-3], являющихся, по сути, квантовыми точками. Рассматриваются как графеновые наноленты [1,2], так и квадратные островки [3]. Следует подчеркнуть, что начало исследований краевых состояний углеродных структур положили работы, относящиеся к кромкам графита (см. [4,5] и приведенные там ссылки). Отметим, что сходные задачи возникают и при изучении контактов латеральных графеноподобных структур, в частности, графена с двумерным гексагональным нитридом бора (h-BN) [6-10]. Во всех цитированных работах отмечается, что особый интерес представляют зигзагообразные края, электронный спектр которых характеризуется плоской зоной и пиком плотности состояний в точке Дирака, что свидетельствует, в частности, о возможности магнитного упорядочения (см. ссылки в [2]).

Ясно, однако, что для реальной приборной графеновой структуры необходима подложка, наличие которой в [1-3] полностью игнорируется. В настоящей работе мы рассмотрим эпитаксиальный графен (эпиграфен) и взаимодействующую с его зигзагообразной кромкой адсорбированную частицу (адчастицу). При этом для описания эпиграфена будет применяться адсорбционная модель $[11,12]$, а для определения электронной структуры его зигзагообразной кромки будут использованы максимально простые способы (метод Калкстейна-Совена и кластерная модель). Отметим, что с точки зрения теории, проблема декорирования островка эпиграфена сводится к задаче о связи чужеродной частицы не только с его граничными атомами, но и с подлож- кой. В качестве подложки рассматривается металл на том, в первую очередь, основании, что существующие в настоящее время латеральные гетероструктуры графен-h-BN сформированы только на $\mathrm{Cu}(111)$ [7-9]. Не следует забывать и о металлических контактах к графену (например, $\mathrm{Cu} / \mathrm{Au}, \mathrm{Ni} / \mathrm{Au}$ и т.д.), являющихся элементом любого прибора. И, наконец, с теоретической точки зрения, рассмотреть влияние металлической подложки на электронные состояния адсорбированного монослоя гораздо проще, чем для полупроводниковой подложки.

\section{2. Метод Калкстейна-Совена}

Закон дисперсии для бесконечного листа свободного графена (рис. 1) в низкоэнергетическом приближении [11-13] можно представить в виде

$$
E_{ \pm}(\mathbf{k})=\varepsilon \pm \xi(\mathbf{k})
$$

Здесь $\varepsilon$ - энергия точки Дирака, в дальнейшем принимаемая за начало отсчета энергии; $\mathbf{k}=\left(k_{x}, k_{y}\right)-$ волновой вектор, отсчитываемый от волнового вектора точки Дирака $\mathbf{K}=(2 \pi / 3 a \sqrt{3}, 2 \pi / 3 q)$ зоны Бриллюэна, ${ }^{1} a$ - расстояние между ближайшими соседями (б.с.); $\xi=\sqrt{\xi_{x}^{2}+\xi_{y}^{2}}, \xi_{x(y)}=(3 t a / 2) k_{x(y)}, t$ - интеграл перехода между б.с.; верхний знак в (1) отвечает зоне проводимости графена, нижний - валентной зоне. Функцию Грина бесконечного листа эпиграфена $\bar{G}(\omega, \xi)=(\omega \mp \xi+i \Gamma)^{-1}$, где $\omega-$ энергия, $\Gamma-$ полуширина квазиуровня адатома углерода $[11,12]$, предста-

\footnotetext{
${ }^{1}$ Отметим: здесь оси $x$ и $y$, изображенные на рис. 1 , соответствуют осям $y$ и $x$ рис. 2 работы [13]; то же относится и к компонентам волнового вектора $k_{x}$ и $k_{y}$.
} 


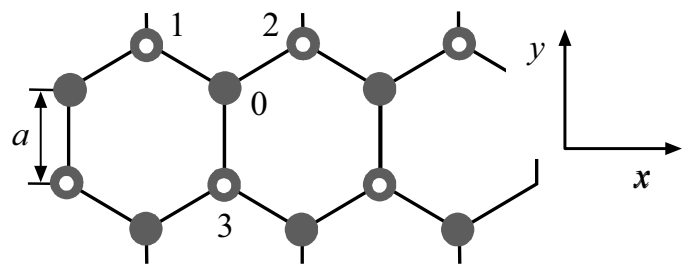

Рис. 1. Структура графена. Обозначенные по-разному атомы типа 0 и атомы типа 1 относятся к различным подрешеткам.

вим в виде $\bar{G}(\omega, \xi)=\operatorname{Re} \bar{G}(\omega, \xi)+i \operatorname{Re} \bar{G}(\omega, \xi)$, где

$$
\begin{aligned}
\operatorname{Re} \bar{G}(\omega, \xi)= & \operatorname{sgn}(\omega) \frac{\omega-\xi}{(\omega-\xi)^{2}+\Gamma^{2}} \\
& +\operatorname{sgn}(-\omega) \frac{\omega+\xi}{(\omega+\xi)^{2}+\Gamma^{2}},
\end{aligned}
$$

$\operatorname{Im} \bar{G}(\omega, \xi)=-\Gamma\left(\frac{\operatorname{sgn}(\omega)}{(\omega-\xi)^{2}+\Gamma^{2}}+\frac{\operatorname{sgn}(-\omega)}{(\omega+\xi)^{2}+\Gamma^{2}}\right)$.

Для образования зигзагообразной кромки разрежем структуру, изображенную на рис. 1 , вдоль оси $x$, проходящей через центры связей типа 0-3. Для математического описания этой процедуры воспользуемся методом Калкстейна и Совена $[14,15]$. Будем считать, что полубесконечной структуре отвечает гамильтониан $H=\bar{H}+T$, где $\bar{H}-$ гамильтониан бесконечного листа графена, $T$ - возмущение, описывающее разрыв $\pi$-связей адатомов углерода вдоль линии разреза. При этом отличными от нуля являются только матричные элементы $T(-1,0)$ и $T(0,-1)$, описывающие связь верхней полубесконечной структуры (с номерами атомных рядов $m \geq 0)$ с нижней полубесконечной структурой $(m \leq-1)$ и равные по модулю $t$. Исходя из уравнения Дайсона $G=\bar{G}+\bar{G} T G$, получим для функции Грина $G$ адатома углерода, принадлежащего верхней кромке $(m=0)$, следующее выражение $[14,15]$ :

$$
G\left(\omega, \xi_{y}\right)=\frac{G_{0}\left(\omega, \xi_{y}\right)}{1-i G_{1}\left(\omega, \xi_{y}\right)},
$$

где

$$
G_{m}\left(\omega, \xi_{y}\right)=N_{x}^{1} \sum_{k_{x}} \exp \left(i m k_{x} a / 2\right) \bar{G}(\omega, \xi)
$$

индекс 0 (1) относится к адатомам углерода типа 0 (адатомам 1 и 2) (рис. 1). Оставаясь в рамках низкоэнергетического приближения [11], т. е. сохраняя только линейные по $\xi$ члены, имеем $G_{1}\left(\omega, \xi_{y}\right) \approx G_{0}\left(\omega, \xi_{y}\right)$. Полагая $G_{0}\left(\omega, \xi_{y}\right)=J_{1}\left(\omega, \xi_{y}\right)+i J_{2}\left(\omega, \xi_{y}\right)$, получим

$$
\begin{aligned}
& \operatorname{Re} G\left(\omega, \xi_{y}\right)=\frac{J_{1}-t\left(J_{1}^{2}+J_{z}^{2}\right)}{\left(1-t J_{1}\right)^{2}+t^{2} J_{2}^{2}}, \\
& \operatorname{Im} G\left(\omega, \xi_{y}\right)=\frac{J_{2}}{\left(1-t J_{1}\right)^{2}+t^{2} J_{2}^{2}},
\end{aligned}
$$

где функции $J_{1}\left(\omega, \xi_{y}\right)$ и $J_{2}\left(\omega, \xi_{y}\right)$ приведены в Приложении, п. 1.
Перейдем теперь непосредственно к рассмотрению декорирования кромки эпиграфена, что сводится к задаче о связи чужеродных адчастиц с этой кромкой. Согласно теории адсорбции [11], функция Грина одиночной (изолированной) адчастицы $G_{a}(\omega)$ определяется выражением

$$
G_{a}^{-1}(\omega)=\omega-\varepsilon_{a}-\Lambda_{a}(\omega)+i \Delta_{a}(\omega) .
$$

Здесь $\varepsilon_{a}-$ энергия работающего на адсорбцию уровня адчастицы, изначально (до адсорбции) заполненного одним электроном, $\Lambda_{a}(\omega)$ и $\Delta_{a}(\omega)-$ функции сдвига и уширения квазиуровня адчастицы, которые в рассматриваемой задаче равны соответственно $V^{2} \operatorname{Re} G(\omega)$ и $-V^{2} \operatorname{Im} G(\omega)$, где $V-$ матричный элемент взаимодействия адчастицы с кромкой эпиграфена,

$$
G(\omega)=2\left(\xi_{y}^{*}\right)^{-1} \int_{-\xi_{y}^{*}}^{\xi_{y}^{*}} G\left(\omega, \xi_{y}\right) d \xi_{y},
$$

где $\xi_{y}^{*}-$ энергия обрезания по $\xi_{y}$. К сожалению, такое интегрирование может быть выполнено только численно даже после достаточно радикальных упрощений, сделанных в п. 1 Приложения. Поэтому, исходя из низкоэнергетического приближения, справедливого лишь для малых энергий в окрестности точки Дирака, и воспользовавшись часто применяемым в теории адсорбции упрощением [11], заменим функции $\Lambda_{a}(\omega)$ и $\Delta_{a}(\omega)$ значениями $\Lambda_{a}(0)=0$ и $\Delta_{a}(0) \equiv \Delta_{0 a}=V^{2} / \Gamma$, так что $G_{a}^{-1}(\omega) \approx \omega-\varepsilon_{a}+i \Delta_{0 a}$. Такой функции Грина отвечает плотность состояний на адчастице (на одну проекцию спина) вида

$$
\rho_{a}(\omega)=\frac{1}{\pi} \frac{\Delta_{0 a}}{\left(\omega-\varepsilon_{a}\right)^{2}+\Delta_{0 a}^{2}} .
$$

Если экстраполировать плотность состояний (7) на всю энергетическую ось, то для числа заполнения квазиуровня адчастицы $n_{a}$ получим следующую оценку:

$$
n_{a}=\frac{2}{\pi} \operatorname{arcctg} \frac{\varepsilon_{a}-\varepsilon_{F}}{\Delta_{0 a}},
$$

где $\varepsilon_{F}-$ энергия Ферми системы. Отсюда приходим к известному результату теории адсорбции [11]: если $\varepsilon_{a}>\varepsilon_{F}$, то заряд адчастицы $Z_{a}=1-n_{a}>0$, в результате чего локальная работа выхода системы в области расположения адчастицы понижается; при $\varepsilon_{a}<\varepsilon_{F}$ имеем $Z_{a}<0$ и соответствующее увеличение работы выхода системы. С качественной точки зрения те же результаты имеют место и при учете взаимодействия между адчастицами при их конечной концентрации [11], что в нашем случае соответствует цепочке, образованной адчастицами, выстроившимися вдоль кромки графена (см. п. 4). 

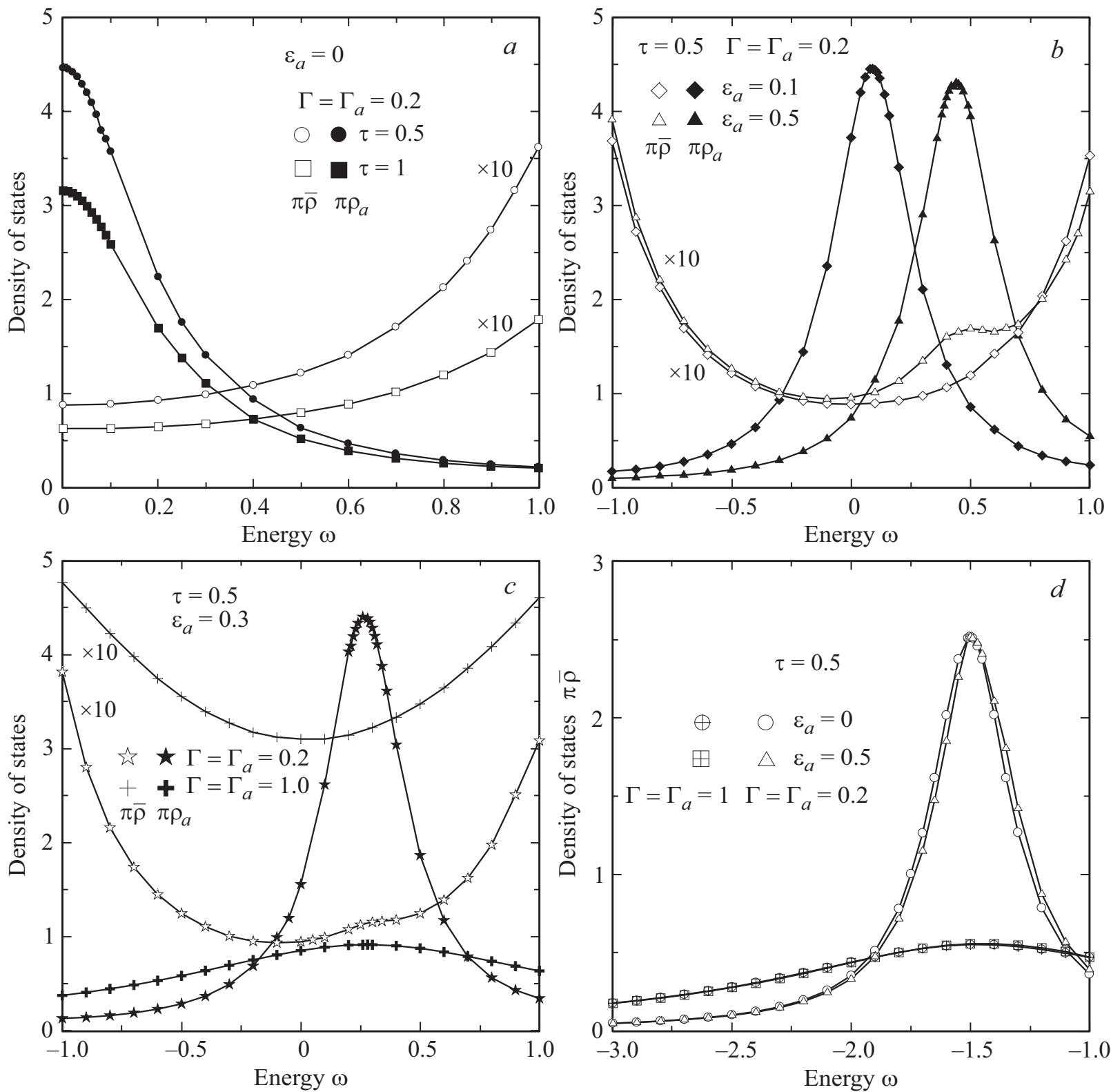

Рис. 2. Плотности состояний на адчастице $\rho_{a}(\omega)$ и связанном с ней адатоме графена $\bar{\rho}(\omega)$ (темные и светлые символы соответственно) для различных значений параметров задачи $\varepsilon_{a}, \tau=\tilde{t} / t, \Gamma=\Gamma_{a}$. Все энергетические величины измеряются в ед. $t$, плотности состояний - в ед. $t^{-1}$. На рис. $2, a$ изображены только правые половины функций $\rho_{a}(\omega)$ и $\bar{\rho}(\omega)$, симметричных относительно $\omega=0$. График функции $\bar{\rho}(\omega)$ увеличен на рисунках $a, b, c$ в 10 раз.

\section{3. Кластерная модель}

Рассмотрим моделирующий зигзагообразную кромку эпиграфена кластер-тример, состоящий из адатомов 0,1 и 2 (рис. 1). Как показано в работе [16] (см. вторую из формул (8)), функция Грина для адатома 0 углерода имеет вид

$$
G(\omega)=g(\omega)\left(1-2 g^{2}(\omega) t^{2}\right)^{-1},
$$

где для металлической подложки функция Грина изолированного (одиночного) адатома углерода есть $g^{-1}=\omega+i \Gamma$ (как и в предыдущем разделе, нуль энер- гии совмещен с точкой Дирака). Легко показать, что функцию Грина (9) можно представить как

$G(\omega)=(g(\omega) / 2)\left((1-g(\omega) t \sqrt{2})^{-1}+(1+g(\omega) t \sqrt{2})\right)$,

так что соответствующая плотность состояний $\rho(\omega)=-\pi^{-1} \operatorname{Im} G(\omega)$ равна

$\rho(\omega)=\frac{\Gamma}{2 \pi}\left(\frac{1}{(\omega+t \sqrt{2})^{2}+\Gamma^{2}}+\frac{1}{(\omega-t \sqrt{2})^{2}+\Gamma^{2}}\right)$,

т.е. представляет собой полусумму двух лоренцевых контуров, центрированных при энергиях $\omega_{ \pm}= \pm t \sqrt{2}$. 
Выражение (10), полученное для адсорбированного тримера, с качественной точки зрения вполне адекватно плотности состояний эпиграфена на металле $[11,17]$. При этом первое и второе слагаемые в скобках отвечают соответственно плотностям состояний валентной зоны и зоны проводимости свободного графена, размытым по энергии вследствие взаимодействия с металлом.

Предположим далее, что адчастица расположена в узле 3 (рис. 1). Включая между „нулевым“ адатомом углерода и декорирующей адчастицей взаимодействие $\tilde{t}$ и воспользовавшись уравнением Дайсона, получим для адчастицы функцию Грина

$$
G_{a}(\omega)=g_{a}(\omega)\left(1-g_{a}(\omega) G(\omega) \tilde{t}^{2}\right)^{-1},
$$

где в случае металлической подложки $g_{a}^{-1}=\omega-\varepsilon_{a}$ $+i \Gamma_{a}$ и $\Gamma_{a}-$ полуширина квазиуровня адчастицы. Общее выражение для плотности состояний на адчастице $\rho_{a}(\omega)=-\pi^{-1} \operatorname{Im} G_{a}(\omega)$ приведено в Приложении, п. 2 , а на рис. 2 представлены зависимости функции $\rho_{a}(\omega)$ от параметров модели. Отметим, что на рис. 2 отображены только случаи, когда $\varepsilon_{a}>0$. Можно показать, что функция $\rho_{a}(\omega)$ инвариантна относительно замены $\varepsilon_{a}$ на $-\varepsilon_{a}$ и $\omega$ на $-\omega$.

На рис. 2, $a, b$ и с отображены некоторые зависимости плотности состояний $\rho_{a}(\omega)$ от параметров задачи. Так, например, при $\varepsilon_{a}=0$ (рис. 2,a) имеем $\pi \rho_{a}(0)=w^{2} /\left(\Gamma_{a} w^{2}+\Gamma \tilde{t}^{2}\right)$, где $w^{2}=2 t^{2}+\Gamma^{2}$ (см. (П5)), откуда следует, что с ростом $\tau=\tilde{t} / t$ плотность состояний $\rho_{a}(\omega)$ понижается. Далее, рис. $2, a, b, c$ показывают, что максимальное значение $\rho_{a}(\omega)$ отвечает энергиям $\omega \sim \varepsilon_{a}$, причем с ростом $Г$ и $\Gamma_{a}$ высота пика уменьшается. При этом, согласно расчетам, можно положить $[a] \approx 0$ (см. (П4)), так что получим

$$
\pi \rho_{a}\left(\varepsilon_{a}\right) \sim\left(\varepsilon_{a}^{2}-w^{2}\right) /\left[\left(\varepsilon_{a}^{2}-w^{2}\right) \Gamma_{a}-\Gamma \tilde{t}^{2}\right] .
$$

Отсюда следует, во-первых, что максимум плотности состояний сравнительно слабо зависит от величины $\varepsilon_{a}$ (рис. 2, $a, b, c)$ и, во-вторых, что с ростом Г и $\Gamma_{a}$ этот максимум понижается (рис. 2,c).

Для оценок числа заполнения адчастицы положим

$$
\rho_{a}(\omega)=\frac{1}{\pi} \frac{\gamma_{a}}{\left(\omega-\varepsilon_{a}\right)^{2}+\gamma_{a}^{2}},
$$

где $\gamma_{a}=\left(\pi \rho_{a}\left(\varepsilon_{a}\right)\right)^{-1}$ и $\rho_{a}\left(\varepsilon_{a}\right)$ дается формулой (12). Число заполнения квазиуровня адчастицы $n_{a}$ есть

$$
n_{a}=\frac{2}{\pi} \operatorname{arcctg} \frac{\varepsilon_{a}-\varepsilon_{F}}{\gamma_{a}}
$$

Заряд адчастицы, содержащей изначально (до адсорбции) один электрон, равен $Z_{a}=1-n_{a}$. При замене $\left(\varepsilon_{a}-\varepsilon_{F}\right)$ на $-\left(\varepsilon_{a}-\varepsilon_{F}\right)$ знак $Z_{a}$ меняется. Если $Z_{a}>0$, частица является донором, если $Z_{a}<0$ - акцептором, $\left|Z_{a}\right|$ определяет переход заряда [11]. При $\Gamma^{2}, \Gamma_{a}^{2}, \varepsilon_{a}^{2} \ll t^{2}$, $\tilde{t}^{2}$ получим $\gamma_{a} \approx \Gamma\left(1+\tau^{2}\right)$. На рис. $3, a$ представлены зависимости $n_{a}\left(\tau^{2}\right)$. С увеличением константы связи $\tau$ плотность состояний $\rho_{a}(\omega)$ расплывается, в результате чего $n_{a}$ увеличивается, а $Z_{a}$ уменьшается. Отметим, что значения $n_{a}$ и $Z_{a}$ при $\tau=0$ относятся к частице, адсорбированной на металле.

Рассмотрим теперь влияние адчастицы на электронное состояние адатома углерода, принадлежащего кромке эпиграфена, которому отвечает функция Грина

$$
\bar{G}(\omega)=G(\omega)\left(1-g_{a}(\omega) G(\omega) \tilde{t}^{2}\right)^{-1}
$$

Соответствующая (15) плотность состояний $\bar{\rho}(\omega)=$ $=-\pi^{-1} \operatorname{Im} \bar{G}(\omega)$ может быть сведена к выражению $(П 7)$ (Приложение, п. 2). Графики $\bar{\rho}(\omega)$ представлены на рис. 2. Основные особенности зависимости $\bar{\rho}(\omega)$ состоят в следующем. Во-первых, имеется ненулевой и сдвинутый относительно точки Дирака $\omega=0$ минимум (рис. 2, $a, b, c),{ }^{2}$ величина которого возрастает с увеличением $\Gamma$ и $\Gamma_{a}$. Так, например, при $\omega=\varepsilon_{a}=0$ и $\Gamma=\Gamma_{a}$ имеем $\pi \bar{\rho}(0)=\Gamma /\left(w^{2}+\tilde{t}^{2}\right)$, так что с ростом $\tilde{t}$ плотность состояний убывает (рис. 2,a). Во-вторых, при $\varepsilon_{a} \neq 0$ в области $\omega \sim \varepsilon_{a}$ наблюдается плато (рис. 2, $b, c){ }^{3}$ которое можно представить как результат наложения плотностей состояний эпиграфена (10) и изолированной адчастицы $\rho_{a}^{0}(\omega)=\left(\Gamma_{a} / \pi\right)\left[\left(\omega-\varepsilon_{a}\right)^{2}+\Gamma_{a}^{2}\right]^{-1}[11]$. В третьих, положение боковых пиков для рассмотренных нами значений параметров почти не зависит от энергии $\varepsilon_{a}$, а их высота уменьшается с ростом $\Gamma=\Gamma_{a}$ (рис. $\left.2, d\right)$. Напомним, что понижение боковых пиков с ростом $\Gamma$ характерно для идеального (без дефектов и адсорбатов) бесконечного листа графена $[11,17]$. Энергии центров правого и левого боковых максимумов $\bar{\rho}(\omega)$ есть $\bar{\omega}_{ \pm} \approx \pm \sqrt{2 t^{2}+\tilde{t}^{2}}$, так что при $\tau=0.5$ имеем $\bar{\omega}_{ \pm} \approx \pm 1.5 t$ (рис. $2, d$ ), что хорошо коррелирует с энергиями $\omega_{ \pm}= \pm \sqrt{2} t$ этих максимумов в отсутствие адчастицы (см. формулу (10)).

Для оценки числа заполнения адатома кромки эпиграфена, связанного с адчастицей, удобно представить плотность состояний $\bar{\rho}(\omega)$ в виде (П8). Тогда, как показано в п. 2 Приложения, для случаев $\Gamma=\Gamma_{a}$ и $\left|\varepsilon_{a}\right|=0-0.5$ имеем

$$
\bar{n}=\frac{1}{\pi}\left(\operatorname{arcctg} \frac{\bar{\omega}_{+}-\varepsilon_{F}}{\Gamma}+\operatorname{arcctg} \frac{\bar{\omega}_{-}-\varepsilon_{F}}{\Gamma}\right),
$$

где $\bar{\omega}_{ \pm}= \pm \sqrt{2 t^{2}+\tilde{t}^{2}}$. Легко видеть, что при $\varepsilon_{F}=0$ получаем $\bar{n}\left(\tau^{2}\right) \equiv 1$. Зависимости $\bar{n}\left(\tau^{2}\right)$ для трех значений $\varepsilon_{F}>0$ представлены на рис. $3, b$. При этом $\bar{n}>1$, так что адатом графена приобретает отрицательный заряд $\bar{Z}=1-\bar{n}$. С ростом $\tau$ заряд адатома графена понижается. При замене $\varepsilon_{F}$ на $-\varepsilon_{F}$ знак $\bar{Z}$ меняется.

\footnotetext{
2 Отметим, что конечное значение функции $\bar{\rho}(0)$ является стандартной характеристикой эпиграфена на металлической подложке $[11,16,17]$.

3 В масштабе, использованном на рис. 2, $a$, а также рис. 2, $b$ при $\varepsilon_{a}=0,1$, плато не проявляется.
} 

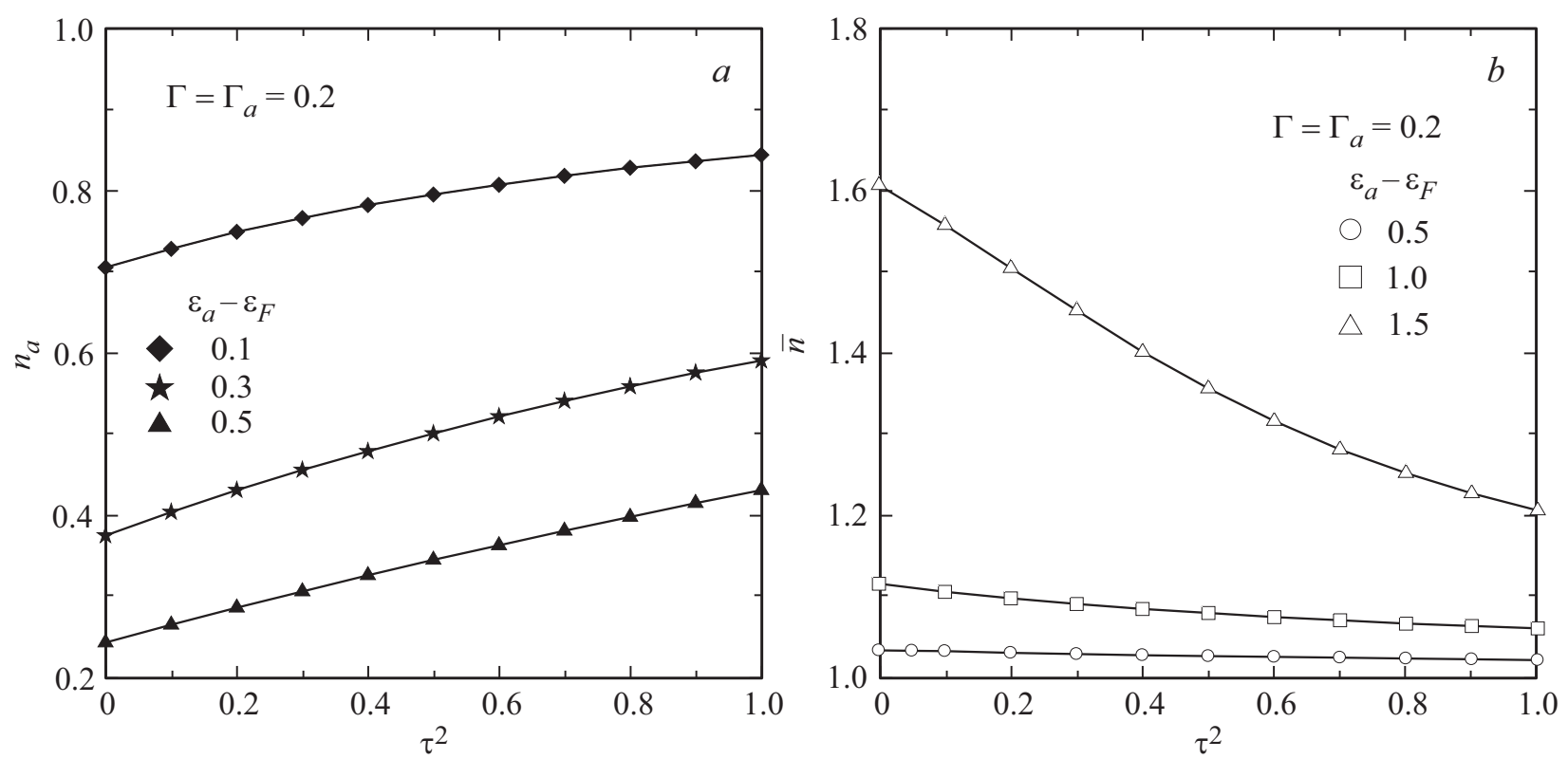

Рис. 3. Зависимости чисел заполнения адчастицы $n_{a}$ и связанного с ней адатома графена $\bar{n}$ (темные и светлые символы соответственно) от квадрата безразмерной константы связи $\tau^{2}$.

\section{4. Обсуждение результатов и заключение}

До сих пор мы рассматривали изолированную декорирующую частицу. Если, однако, вдоль кромки эпиграфена выстраивается цепочка взаимодействующих друг с другом адчастиц, их заряд, естественно, изменяется [11]. Если каждый адатом зигзагообразной кромки связан с адчастицей, то будем говорить о степени покрытия кромки адчастицами $\Theta=1$. При этом расстояние между ближайшими адчастицами равно $c_{0}=a \sqrt{3}$ (рис. 1). Если же только каждый второй адатом кромки взаимодействует с адчастицей, то $\Theta=1 / 2$ и т.д. Считаем, таким образом, что адчастицы образуют эквидистантную цепочку с расстоянием между б.с., равным $c=c_{0} / \Theta$. Если адчастицы заряжены, то, учитывая их заряды изображения в металлической подложке, следует говорить о цепочке параллельных диполей. Считая диполи точечными, можно показать, что диполь-дипольное отталкивание между ними приводит к смещению квазиуровня $\varepsilon_{a}$ в положение

$$
\varepsilon_{a}^{\prime}=\varepsilon_{a}-\xi_{\text {dip }} \Theta^{3} Z_{a}(\Theta),
$$

где константа диполь-дипольного отталкивания $\xi_{\text {dip }}=$ $=2 e^{2} l^{2} A^{\prime} / c_{0}^{3}, Z_{a}(\Theta)$ - зависящее от степени покрытия значение заряда адчастицы, $e-$ величина заряда электрона, $2 l$ - плечо диполя,

$$
A^{\prime}=2 \sum_{n=1}^{\infty} n^{-3}=\zeta(3)=2.40
$$

и $\zeta(. .$.$) - дзета-функция Римана. Заменяя в выражени-$ ях (8) и (14) $\varepsilon_{a}$ на $\varepsilon_{a}^{\prime}$, приходим к самосогласованному уравнению

$$
Z_{a}(\Theta)=\frac{2}{\pi} \operatorname{arctg} \frac{\varepsilon_{a}-\varepsilon_{F}-\xi_{\mathrm{dip}} \Theta^{3} Z_{a}(\Theta)}{\Gamma_{a}}
$$

где $\Gamma_{a}$ есть $\Delta_{0 a}$ и $\gamma_{a}$ в случаях выражений (8) и (14) соответственно. Отметим, что уравнение (18) является одномерным аналогом результата теории дипольдипольного взаимодействия для двумерной решетки адатомов, где вместо $\Theta^{3}$ в $(18)$ входит $\Theta^{3 / 2}$ и $A^{\prime} \sim 10$ [18] (см. также $[11,19])$.

Зависимости $Z_{a}(\Theta)$, представленные на рис. 4, $a$, типичны для адсорбционных систем и демонстрируют деполяризацию (уменьшение величины заряда адчастицы) с ростом степени покрытия $[11,19,20]$. Отметим три характерных участка этой зависимости: 1) малые покрытия $(\Theta \leq 0.1-0.2)$, заряд $Z_{a}(\Theta) \sim Z_{a}(0)$ и меняется слабо; 2) промежуточные покрытия $(0.2<\Theta \leq 1)$, заряд быстро спадает с ростом $\Theta ; 3)$ высокие покрытия $(0.5<\Theta \leq 1)$, спад заряда замедляется. С увеличением $\varepsilon_{a}-\varepsilon_{F}$ и уменьшением $\Gamma_{a}$ зависимость $Z_{a}(\Theta)$ обостряется.

Перейдем теперь к обсуждению изменения работы выхода в области зигзагообразного контакта $\Delta \phi$, вызванного адсорбцией частиц. Здесь непосредственная экстраполяция результатов работы [18] на рассматриваемую нами структуру затруднена в силу ее неоднородности. ${ }^{4}$ Тем не менее, можно утверждать, что $\Delta \phi \propto-Z_{a}(\Theta) \Theta$.

\footnotetext{
${ }^{4}$ Вопрос о неоднородной, „пятнистой“ поверхности поднимался, однако, еще в героический период эмиссионной электроники $[21,22]$. Под „Пятном“ понимается неоднородность типа островка адатомов или выхода „неправильной“ грани на поверхность монокристалла. Возникает также вопрос о минимальной величине островка, „замечаемого“ эмитируемыми электронами [23].
} 

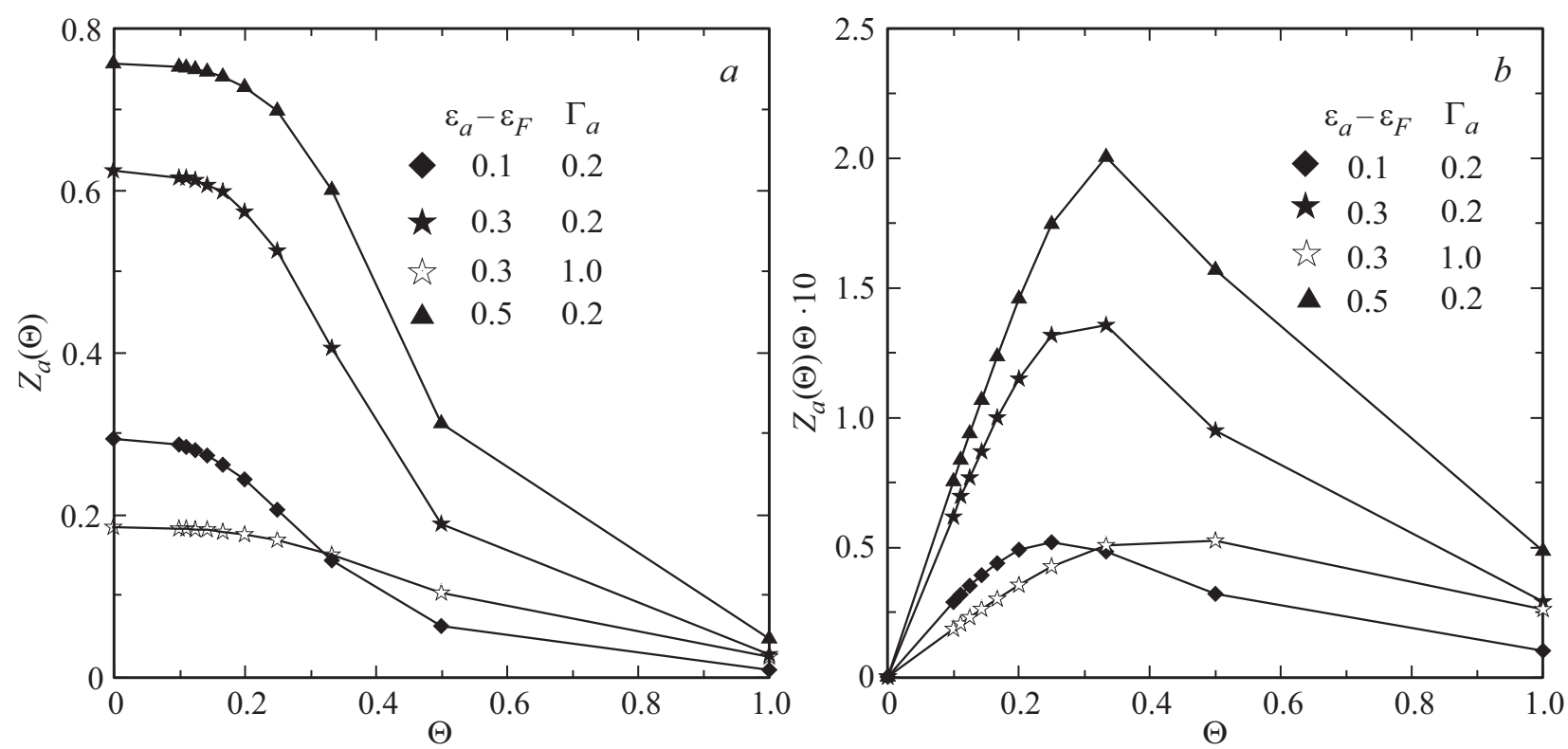

Рис. 4. Зависимости заряда адчастицы $Z_{a}(a)$ и характеризующего изменение работы выхода произведения $Z_{z}(\Theta) \Theta(b)$ от степени покрытия графеновой кромки адчастицами $\Theta$ при $l=c_{0}=a \sqrt{3}=2.46 \AA$ и $\xi_{\text {dip }}=10$ (в ед. $t$ ). Линии на рисунках носят вспомогательный характер, физический смысл имеют точ- ки, отмеченные символами. Эти точки отвечают эквидистантной цепочке адчастиц с расстоянием между ближайшими соседями $c=N c_{0}$, где $N=\infty, 10,9, \ldots, 2,1$, или степеням покрытия $\Theta=0,1 / 10,1 / 9, \ldots, 1 / 2,1$.

Зависимости $Z_{a}(\Theta) \Theta$, представленные на рис. $4, b$, качественно совпадают с экспериментально наблюдаемыми зависимостями $\Delta \phi(\Theta)$ [24]. С ростом $\varepsilon_{a}-\varepsilon_{F}$ функция $\Delta \phi(\Theta)$ обостряется, а ее максимум сдвигается в сторону больших покрытий. Увеличение $\Gamma_{a}$ сглаживает зависимость $\Delta \phi(\Theta)$. Отметим, что приведенные оценки относятся к работе выхода электрона из металлической подложки в полосе, окружающей цепочку взаимодействующих с кромкой адчастиц,

В работе [1] теоретически исследовалась функционализация свободной графеновой наноленты за счет присоединения к одной из ее зигзагообразных кромок ряда органических групп (кетона, гидроксила, карбоксила, карбонила), а к другой - водорода. Расчеты проводились в рамках теории функционала плотности. При этом в непосредственную связь с атомом кромки графена вступали атомы О (кетон и гидроксил) и С (карбонил и карбоксил). Аналогичным образом в [2] в качестве декорирующих частиц рассматривался водород. К сожалению, подложка в $[1,2]$ не учитывалась, так что сравнение с нашими результатами не представляется возможным. Следует добавить, что расчеты $[1,2]$ дают информацию о работе выхода электрона из глубины наноленты через двойной электрический слой, возникающий на ее кромках вследствие декорирования.

Поясним теперь, на каком основании мы в расчетах принимали, для простоты, что $\Gamma=\Gamma_{a}$. В задаче об адсорбции Андерсона-Ньюнса $[11,19,24]$ полуширина квазиуровня адатома $\Gamma=\pi V_{c s}^{2} \rho_{\text {sub }}$, где $V_{c s}-$ матричный элемент взаимодействия атом углерода - подложка, $\rho_{\text {sub }}$ - плотность состояний подложки, в простейшем случае аппроксимируемая для металла константой. Будем оценивать $V_{c s}$ двухцентровым интегралом, отвечающим связи атома подложки с атомом углерода. Если воспользоваться методом связывающих орбиталей Харрисона [25], то $V_{c s}=\eta_{c s}\left(\hbar^{2} / m_{e} d^{2}\right)$, где $\hbar-$ приведенная постоянная Планка, $m_{e}$ - масса электрона, $d_{c s}-$ расстояние между центрами атомов углерода и подложки, принимаемое равным сумме атомных радиусов, и $\eta_{c s}-$ численный множитель, определяемый характером связи $(\sigma$ или $\pi)$ и орбиталями, в этой связи участвующими. Аналогично, для адчастицы имеем $\Gamma_{a}=\pi V_{a s}^{2} \rho_{\text {sub }}$. Полагая $d_{c s}=d_{a s}$ и пренебрегая различием $\eta_{c s}$ и $\eta_{a s}$ (см. сноску 4), получаем $\Gamma=\Gamma_{a}$.

Итак, в настоящей работе мы использовали два подхода к задаче о декорировании. Первый подход основан на схеме Калкстейна-Совена в приближении сильной связи. Это схема последовательна и логична, но, к сожалению, не позволяет простым образом получить аналитические выражения для перехода заряда между адчастицами и зигзагообразной кромкой эпиграфена. Второй подход состоит в использовании простого кластера-тримера, моделирующего фрагмент зигзагообразной кромки. Такая модель позволяет получить простые аналитические оценки. Интересно отметить, что та же ситуация имеет место для гетероконтакта латеральных структур [10].

Здесь мы ограничились рассмотрением только металлической подложки. В принципе, не намного сложнее рассмотреть и полупроводниковую подложку (см. $[26,27])$. При этом, однако, возникает большое число возможных вариантов результирующей плотности состо- 
яний системы. Так как в настоящее время какие либо латеральные гетероструктуры на таких подложках не сформированы, постановка подобной задача, по нашему мнению, преждевременна.

\section{Приложение}

1. Интегрируя выражения (2) и (3) в виде $2\left(\xi_{x}^{*}\right)^{-1} \int_{-\xi_{x}^{*}}^{\xi^{*}}(\ldots) d \xi_{x}$, где $\xi_{x}^{*}-$ энергия обрезания по $\xi_{x}[11]$, получим

$$
\begin{aligned}
& J_{1}\left(\omega, \xi_{y}\right) \approx \frac{1}{\left(\omega^{2}+\Gamma^{2}\right)}\left(\omega+\frac{\operatorname{sgn}(\omega)}{2} \frac{\omega^{2}-\Gamma^{2}}{\omega^{2}+\Gamma^{2}} A\left(\xi_{y}\right)\right), \\
& J_{2}\left(\omega, \xi_{y}\right) \approx-\frac{\Gamma}{\omega^{2}+\Gamma^{2}}\left(1+\frac{|\omega|}{\left(\omega^{2}+\Gamma^{2}\right)} A\left(\xi_{y}\right)\right), \\
& A\left(\xi_{y}\right)=\sqrt{\left(\xi_{x}^{*}\right)^{2}+\xi_{y}^{2}}+\frac{\xi_{y}^{2}}{\xi_{x}^{*}} \ln \frac{\xi_{x}^{*}+\sqrt{\left(\xi_{x}^{*}\right)^{2}+\xi_{y}^{2}}}{\xi_{y}} .
\end{aligned}
$$

Отсюда следует, что плотность состояний $\rho\left(\omega, \xi_{y}\right)=-\pi^{-1} J_{2}\left(\omega, \xi_{y}\right)$ имеет максимум, равный $(\pi \Gamma)^{-1}$ при $\omega=0$, т. е. в точке Дирака. В этой же точке функция $J_{2}\left(\omega, \xi_{y}\right)$ испытывает скачок от $+\left(A\left(\xi_{y}\right) / \Gamma\right)$ до $-\left(A\left(\xi_{y}\right) / \Gamma\right)$, что является следствием приближенного интегрирования.

Для порядковой оценки выражений (П1) и (П2) положим в (ПЗ) $\xi_{y}=\xi_{y}^{*}=\xi^{*} / \sqrt{2}$, где $\xi^{*}=\sqrt{\left(\xi_{x}^{*}\right)^{2}+\left(\xi_{y}^{*}\right)^{2}}$. Отсюда получаем $A\left(\xi_{y}^{*}\right) \sim 1$. Тогда (П1) и (П2) переходят в оценочные выражения для $J_{1}(\omega)$ и $J_{2}(\omega)$.

2. Плотность состояний на адчастице $\rho_{a}(\omega)=-\pi^{-1} \operatorname{Im} G_{a}(\omega)$ дается выражением

$$
\rho_{a}(\omega)=\frac{1}{\pi} \frac{[b][c]-[a][d]}{[a]^{2}+[b]^{2}}
$$

где

$$
\begin{gathered}
{[a]=\left(\omega^{2}-w^{2}\right)\left(\omega-\varepsilon_{a}\right)-\omega \tilde{w}^{2},} \\
{[b]=\left(\omega^{2}-w^{2}\right) \Gamma_{a}+2 \omega\left(\omega-\varepsilon_{a}\right) \Gamma-\Gamma \tilde{t}^{2},} \\
{[c]=\omega^{2}-w^{2}, \quad[d]=2 \omega \Gamma,}
\end{gathered}
$$

где $w^{2}=2 t^{2}+\Gamma^{2}, \quad \tilde{w}^{2}=2 \Gamma \Gamma_{a}+\tilde{t}^{2}$. В точке Дирака имеем

$$
\rho_{a}(0)=\frac{1}{\pi} \frac{w^{2}\left(\Gamma_{a} w^{2}+\Gamma \tilde{t}^{2}\right)}{\varepsilon_{a}^{2} w^{4}+\left(\Gamma_{a} w^{2}+\Gamma \tilde{t}^{2}\right)^{2}} .
$$

Знак энергии уровня адчастицы $\varepsilon_{a}$ не влияет на величину $\rho_{a}(0)$, что объясняется симметричностью плотности состояний $\rho(\omega)=-\pi^{-1} \operatorname{Im} G(\omega)$ относительно $\omega=0$ (см. рис.1, $b$ работы [16]). При выполнении условий $\Gamma_{a} \sim \Gamma \ll \tilde{t} \sim t,\left|\varepsilon_{a}\right|$ получаем $\rho_{a}(0) \sim \Gamma / \pi \varepsilon_{a}^{2}$. Отметим, что даже в простейшем случае $\Gamma_{a}=\Gamma=\tilde{t}=t, \varepsilon_{a}=0$ выражение для $\rho_{a}(\omega)$ является достаточно громоздким:

$$
\rho_{a}(\omega)=\frac{\Gamma}{\pi} \frac{\omega^{4}+5 \omega^{2} \Gamma^{2}+12 \Gamma^{4}}{\omega^{2}\left(\omega^{2}-6 \Gamma^{2}\right)^{2}+\Gamma^{2}\left(3 \omega^{2}-4 \Gamma^{2}\right)^{2}} .
$$

Плотность состояний $\bar{\rho}(\omega)=-\pi^{-1} \operatorname{Im} \bar{G}(\omega)$ на адатоме графена, связанном с адчастицей (соответствующая функции Грина (15)), может быть представлена в виде

$$
\bar{\rho}(\omega)=\frac{1}{\pi} \frac{[b]\left[c^{\prime}\right]-[a]\left[d^{\prime}\right]}{[a]^{2}+[b]^{2}},
$$

где $\left[c^{\prime}\right]=\omega\left(\omega-\varepsilon_{a}\right)-\Gamma \Gamma_{a}$ и $\left[d^{\prime}\right]=\left(\omega-\varepsilon_{a}\right) \Gamma+\omega \Gamma_{a}$. При $\Gamma=\Gamma_{a}$ получим следующие функции $\bar{\rho}(\omega)$ :

$$
\begin{gathered}
\bar{\rho}(0)=\frac{\Gamma}{\pi} \frac{\Gamma^{2}\left(w^{2}+\tilde{t}^{2}\right)+\varepsilon_{a}^{2} w^{2}}{\Gamma^{2}\left(w^{2}+\tilde{t}^{2}\right)^{2}+\varepsilon_{a}^{2} w^{4}}, \\
\bar{\rho}\left(\varepsilon_{a}\right)=\frac{\Gamma}{\pi} \frac{\varepsilon_{a}^{2} \tilde{w}^{2}-\Gamma^{2}\left(\varepsilon_{a}^{2}-w^{2}-t^{2}\right)}{\varepsilon_{a}^{2} \tilde{w}^{4}+\Gamma^{2}\left(\varepsilon_{a}^{2}-w^{2}-t^{2}\right)^{2}},
\end{gathered}
$$

где $\tilde{w}^{2}=\left(2 \Gamma^{2}+\tilde{t}^{2}\right)$. К значимым характеристикам относятся также высоты боковых максимумов $\bar{\rho}_{ \pm}=\bar{\rho}\left(\omega_{ \pm}\right)$, отвечающих энергиям $\omega_{ \pm}$.

Для оценки числа заполнения адатома графена, взаимодействующего с адчастицей, удобно представить плотность состояний $\bar{\rho}(\omega)$ в виде, аналогичном выражению (10). Положим поэтому

$$
\begin{aligned}
\bar{\rho}^{*}(\omega)= & \frac{\Gamma^{*}}{2 \pi}\left(\frac{1}{\left(\omega-\varepsilon^{*}+t^{*}\right)^{2}+\Gamma^{* 2}}\right. \\
& \left.+\frac{1}{\left(\omega-\varepsilon^{*}-t^{*}\right)^{2}+\Gamma^{* 2}}\right),
\end{aligned}
$$

где $\Gamma^{*}, t^{*}$ и $\varepsilon^{*}-$ параметры. Легко показать, что центральный минимум функции (П10), имеющий место при $\omega=\varepsilon^{*}$, равен

$$
\bar{\rho}^{*}\left(\varepsilon^{*}\right)=\frac{1}{\pi} \frac{\Gamma^{*}}{t^{* 2}+\Gamma^{* 2}} .
$$

Полагая $\left|\omega-\varepsilon^{*}\right| \ll t^{*}$, получим

$$
\bar{\rho}^{*}(\omega) \approx \frac{1}{\pi} \frac{\Gamma^{*}}{t^{* 2}+\Gamma^{* 2}}\left(1+\frac{4 t^{* 2}\left(\omega-\varepsilon^{*}\right)^{2}}{\left(t^{* 2}+\Gamma^{* 2}\right)^{2}}\right) .
$$

При $\varepsilon^{*}<0$ имеем $\bar{\rho}^{*}(\omega<0)>\bar{\rho}^{*}(\omega>0)$, что соответствует результатам, представленным на рис. $2, b$ и $c$. В пределе $\left|\omega-\varepsilon^{*}\right| \gg t^{*}, \quad \Gamma^{*}, \quad\left|\varepsilon^{*}\right|$ получаем $\bar{\rho}^{*}(\omega) \sim \Gamma^{*} / \pi \omega^{2}$ (рис. $2, d$ ).

В общем случае получить аналитические выражения для связи параметров $\Gamma^{*}, t^{*}, \varepsilon^{*}$ с исходными параметрами задачи $\Gamma, \Gamma_{a}, \varepsilon_{a}, t$ и $\tilde{t}$ возможным не представляется. Рассмотрим поэтому квазисвободный эпиграфен и слабо связанную с подложкой адчастицу, полагая $\Gamma^{2}=\Gamma_{a}^{2} \ll t^{2}$. Как показали расчеты, представленные на рис. 2 для $\Gamma=\Gamma_{a}=0.2$, при $\bar{\omega}_{ \pm} \approx \pm 1.5 t$ максимумы плотности состояний $\pi \bar{\rho}\left(\omega_{ \pm}\right) \approx\left(\left[c^{\prime}\right] /[b]\right)_{\omega_{ \pm}}$, так как $[a]_{\omega_{ \pm}} \approx 0$. Действительно, после перехода к безразмерным единицам последнее условие есть

$$
\left(\omega_{ \pm}^{2}-w^{2}\right)\left(\omega_{ \pm}-\varepsilon_{a}\right)-\omega_{ \pm}\left(2 \Gamma^{2}+\tau^{2}\right) \approx 0,
$$

где $w^{2}=2+\Gamma^{2}$. В нулевом приближении по $\Gamma^{2}$ для $\varepsilon_{a}=0$ получаем $\bar{\omega}_{ \pm} \approx \pm \sqrt{2+\tau^{2}}$, откуда при 
$\tau=0.5$ имеем $\bar{\omega}_{ \pm} \approx \pm 1.5$. Для $\varepsilon a \neq 0$ получаем поправку порядка $\left(2-\omega_{ \pm}^{2}\right) \varepsilon_{a}$, которая для использованного нами максимального значения $\varepsilon_{a}=0.5$ равна 0.125. Поэтому при выполнении неравенства $\left|\varepsilon_{a}\right| \ll t$ в выражении (П10) положим $\varepsilon^{*}=0$ и $t^{*} \approx \sqrt{2+\tau^{2}}$. Так как $\pi \bar{\rho}\left(\bar{\omega}_{ \pm}\right) \approx\left(2 \Gamma^{*}\right)^{-1}$, имеем $\pi \bar{\rho}\left(\omega_{ \pm}\right) \approx\left(\left[c^{\prime}\right] /[b]\right)_{\omega_{ \pm}} \approx 2.5$ (см. рис. $\left.2, d\right)$, откуда $\Gamma^{*} \approx 0.2$. Тогда $\pi \bar{\rho}^{*}(0) \approx \Gamma^{*} /\left(2+\tau^{2}\right) \sim 0.1$, что хорошо согласуется с $\bar{\rho}(0) \approx w^{-2} \sim 0.1$ и результатами, представленными на рис. $2, a, b, c$.

\section{Список литературы}

[1] R. Taira, A. Yamanaka, S. Okada. Appl. Phys. Express 9, 115102 (2016).

[2] M.R. Mananghaya, G.N. Santos, D. Yu, C. Stampfl. Sci. Rep. 7, 15727 (2017).

[3] Z.S. Sadeq, R.A. Muniz, J.E. Sipe. Phys. Rev. Mater. 2, 014001 (2018).

[4] K. Nakada, M. Fujita, G. Dresselhaus, M. Dresselhaus. Phys. Rev. B 54, 24, 17954 (1996).

[5] Y. Miyamoto, K. Nakada, M. Fujita. Phys. Rev. B 59, 9858 (1999).

[6] S. Okada, M. Igami, K. Nakada, A. Oshiyama. Phys. Rev. B 62, 9896 (2000).

[7] M.P. Levendorf, C.-J. Kim, L. Brown, P.Y. Huang, R.W. Havener, D.A. Muller, J. Park. Nature 488, 627 (2012).

[8] Z. Liu, L. Ma, G. Shi, W. Zhou, Y. Gong, S. Lei, X. Yang, J. Zhang, J. Yu, K.P. Hackenberg, A. Babakhani, J.-C. Idrobo, R. Vajtai, J. Lou, P.M. Ajayan. Nature Nanotechnol. 8, 119 (2013).

[9] G.C. Loh, R. Pandey. J. Mater. Chem. C 3, 5918 (2015).

[10] С.Ю. Давыдов. Письма в ЖТФ 44, 21, 55 (2018).

[11] С.Ю. Давыдов. Теория адсорбции: метод модельных гамильтонианов. Изд-во СПбГЭТУ „ЛЭТИ“, СПб (2013). 235 c.; twirpx.com/file/1596114/.

[12] С.Ю. Давыдов. ФТТ 59, 1650 (2017).

[13] A.H. Castro Neto, F. Guinea, N.M.R. Peres, R.S. Novoselov, A.K. Geim. Rev. Mod. Phys. 81, 109 (2009).

[14] D. Kalkstein, P. Soven. Surf. Sci. 26, 85 (1971).

[15] С.Ю. Давыдов. ФТТ 60, 1389 (2018).

[16] С.Ю. Давыдов. ФТТ 54, 2193 (2012).

[17] С.Ю. Давыдов. ФТП 47, 97 (2013).

[18] J.P. Muscat, D.M. Newns. J. Phys. C 7, 2630 (1974).

[19] С.Ю. Давыдов, А.А. Лебедев, О.В. Посредник. Элементарное введение в теорию наносистем. Лань, СПб (2014). Гл. 9.

[20] О.М. Браун, В.К. Медведев. УФН 157, 631 (1989).

[21] Л.Н. Добрецов, М.В. Гомоюнова. Эмиссионная электроника. Наука, М. (1966).

[22] Э.Я. Зандберг, Н.И. Ионов. Поверхностная ионизация. Наука, М. (1969).

[23] С.Ю. Давыдов. ЖТФ 49, 211 (1979).

[24] С.Ю. Давыдов, С.В. Трошин. ФТТ 49, 1508 (2007).

[25] W.A. Harrison. Phys. Rev. B 27, 3592 (1983).

[26] С.Ю. Давыдов. ФТТ 58, 779 (2016).

[27] С.Ю. Давыдов. ФТТ 58, 1182 (2016).

Редактор Е.Ю. Флегонтова 